\title{
An Approach of Self-Organizing Systems Based on Factor-Order Space
}

\author{
Jin Li, Ping $\mathrm{He}^{*}$ \\ Department of Information, Liaoning Police College \\ Dalian, 116036 Liaoning, China
}

\begin{abstract}
To explore new system self-organizing theory, it's urgent to find a new method in the system science. This paper combines factor space theory with system non-optimum theory, applies it into the research of system self-organizing theory and proposes new concepts as system factor space, object-factor and space-order relation. It constructs factor-space framework of system self-organizing based on factor mapping and object inversion, studies system ordering from a new perspective with optimum and non-optimum attributes as the basis of system uncertainty, and expands factor space theory from $f(0, \bar{o})$ to $f(o, 0)$. The research suggests that the construction of system factor space is to build an information system capable of selflearning for system self-organization and better enhance functions of system self-organization by adopting information fusion of data analysis and perception judgment.
\end{abstract}

Keywords-Self-organization; factor-order space; extended order; extended entropy; systems level

\section{INTRODUCTION}

From the perspective of general system theory, system is a unity (complex, whole) composed by several interrelated elements (objects). The purpose of any system study is how to exert the function of the system, and to achieve acceptable goal. The main research content of the traditional system theory: determine the running rules and changing rules that fit the characteristics of system, and thus, make the system from disordered structure (random) into a certain behavior and target of ordered structure. The dissipative structure theory of I. Prigogine, the synergy theory of Herman Hake and the mutation theory of $\mathrm{R}$. Thom have established a relatively complete theoretical system, and made a contribution to the development of system science. [1] It is not difficult to find out traditional research of self-organizing theory ignores the factor study which decides the existence and change of the objects. Actually, factors are roots of the existence and development of anything, like the genes of an organism. So, it is worthy of introducing the factor theory into the study of system selforganization. [2]

With the deepening of research on the theory and application of system self-organization, the researchers find out that the order of a system is influenced by related factors with positive and negative attributes, and the recognition and adjustment of different factor attributes is an important content of the system self-organization process. Literature [3] defines positive attribute as optimum attribute, negative attribute as non-optimum attribute. Literature [4] establishes a non- optimum diagnosis model of network system and proposes that non-optimum attribute is the risk source of network system by adopting non-optimum theory to the order of network system. The literature research on relevant information system discovers [5-8] that the order of information system comes from the order of information factors, that is to say, the ordered structure and the mode of an information system is realized in the process of self-organization of factor-space. Under this background, and based on non-optimum and factor space theory [9], this paper proposes is a kind of research method for self-organization from a new system thinking perspective, and introduces a new research content for the theory of factor space. Two main contributions of this paper are: to establish factor space theory of system self-organization; to propose orderly structured evaluation method based on factor-order.

The objective of this paper is to analyze non-optimum problems of the system's self-organization and to discuss a method for evaluating systems level based on the theory of non-optimum analysis. The paper also put forward a new concept called extended entropy with $\lambda$ order (optimum order), $\eta$ order (non-optimum order) and $\lambda-\eta$ order (mesooptimum order).

The remaining parts of the paper are structured as follows: We discuss the basic conception of factor space, as well as the factors level based on factor-order in Section 2. In Section 3 we present a practical notion to describe self-organizing systems based on extended order. Finally, Section 4 concludes the paper and sketches possible further research and applications based on the presented approach.

\section{FACTOR SPACE AND FACTOR ORDER}

\section{A. Non-optimum and Self-organization}

The self-organization system arises during balancing that optimum and non-optimum attributes. Thus, if a system factor has optimum and non-optimum attributes at same time, it is called meso-optimum factor [10]. In traditional selforganization theory, the standards of system order are expressed by optimum attributes of system factor. But it is still difficult to solve the system analysis problem with nonoptimum attributes. The primary cause is uncertainty of optimum attribute and the existence of non-optimum ones. In fact, the process of the self-organization is based on the comparison of optimum and non-optimum attributes.

The diagnosis of the system uncertainty is created by the reversed way of thinking. Therefore, in research self- 
organization system with the uncertainty, we must be considering the optimum and the non-optimum attributes of the system factors, as well as changes of these attributes. In fact, the diagnosis of the system uncertainty is the most important parts of self-organization system. It consists of several processes: (1) Non-optimum attributes identification of selforganization system. (2) Non-optimum attributes evaluation of self-organization system.

\section{B. Basic Conception}

Definition 2.1 Let $P=\left\{p_{1}, p_{2}, \cdots, p_{L}\right\}$ be a projects set of the system, $F=\left\{f_{1}, f_{2}, \cdots, f_{k}\right\}$ be a factors set of the projects, $R(o, \bar{O})$ be a factor relation based on the optimum and the non-optimum attribute, then $\Omega=(P, f, R(o, \bar{O}))$ be called a factor space of the system.

The factor set $F$ of the system is divided into three parts: optimum attribute, non-optimum attribute and unknown attribute. If it is a complete factor space of the system, the measurement of unknown attribute must be equal to zero. Otherwise it is an incomplete factor space of the system. Therefore a complete factor space can be regard as a special case of an incomplete factor space. [8] The basic frame of factor space is shown in Fig. 1.

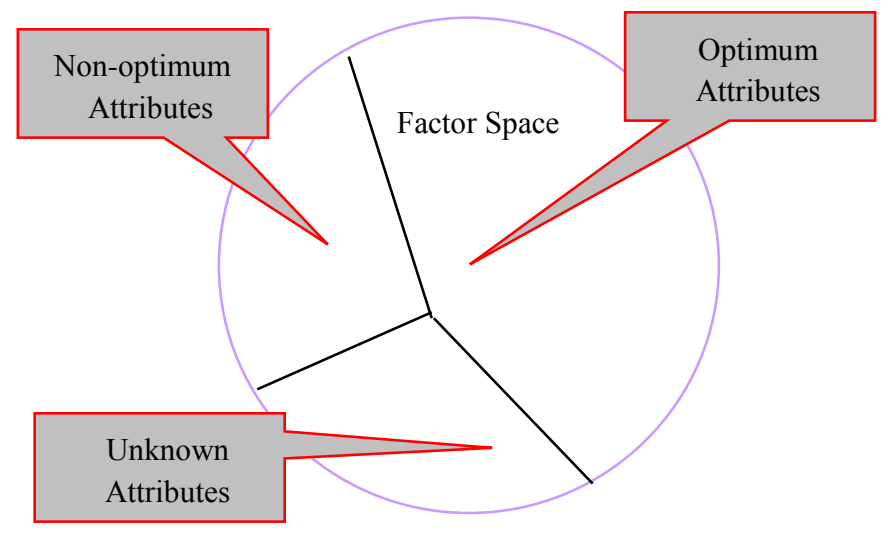

Fig. 1. Incomplete factor space

Definition 2.2 Let $P=\left\{p_{1}, p_{2}, \cdots, p_{L}\right\}$ be a projects set of the system, $F=\left\{f_{1}, f_{2}, \cdots, f_{k}\right\}$ be a factors set of the projects, and $f(o, \bar{o})$ a binary relation defined between $O$ and $\bar{O}$, where $O=\left\{o_{1}, o_{2}, \cdots, o_{n}\right\}$ be a set of the optimum attributes of $F, \bar{O}=\left\{\bar{o}_{1}, \bar{o}_{2}, \cdots, \bar{o}_{n}\right\}$ be a set of the non-optimum attributes of $F$, then there exist to be a set $f(o, \bar{o})=\left\{<o_{i}, \bar{o}_{i}>: o_{i} \in O, \bar{o}_{i} \in \bar{O}\right\}$ where $i=1, \cdots, n$, and $R(o, \bar{o})=f(o, \bar{o})$ be called a relationship of the mesooptimum based on the optimum and the non-optimum attributes.
We use an ordered triad $f(o, \bar{o})$ as the meso-optimum relationship of the factor based on the optimum and nonoptimum attributes for describing system, called the factororder of the system.

\section{The Analysis of Systems Level Based on Factor-Order}

In the research of the self-organization of the uncertain system, the goals of the self-organization are the transformations on the factor level of the system. If the transformations are negative, we can call them friction nonoptimum. If they are positive, we can call them optimum. Thus, meso-optimum relation $R(o, \bar{o})$ is decided by differences the optimum and non-optimum degree and if the results of decisions fall in this trusted classification, we can call them trusted, which decide the optimum degree and the nonoptimum of the system factor.

Definition 2.3 Let $v(o, \bar{o})$ be a value set of factor-order $f(o, \bar{o})$ of the system, then there exist to be a mapping

$$
f_{j}: v(o, \bar{o}) \rightarrow[-1,1], f_{j} \in F,(j=1,2, \cdots, m), \text { where }
$$
the $v(o, 0) \rightarrow[0,1]$, the $v(0, \bar{o}) \rightarrow[-1,0]$ and denote respectively the degree of the positive level, the degree of the negative level, if $v(o, \bar{o}) \rightarrow[-1,1]$ then denote respectively the degree of the neutrally level.

The major function of the self-organization of the system is to optimal the running of the system, develop its goals; they have to experience from minimum non-optimum to maximum optimum, that is

$$
\begin{aligned}
& f_{j}(v(\operatorname{Min}(o), \operatorname{Max}(\bar{o}))) \rightarrow f(o, \bar{o}) \\
& \rightarrow f_{j}(v(\operatorname{Max}(o), \operatorname{Min}(\bar{o}))),
\end{aligned}
$$
that is

and from maximum optimum to minimum non-optimum,

$$
\begin{aligned}
& f(v(\operatorname{Max}(o), \operatorname{Min}(\bar{o}))) \rightarrow f(o, \bar{o}) \\
& \rightarrow f(v(\operatorname{Min}(o), \operatorname{Max}(\bar{o})))
\end{aligned}
$$

If the system is not featured with this attribute, it doesn't need self-organization either. The analysis shows that systems always stay on the border of optimum and non-optimum, that is, $f(v(o, \bar{o}))$ and the aim of self-organization is to bring the system from the satisfaction of the lower levels to the higher level. Of course, the actual angle of the system doesn't have the optimal criteria, and it is also not necessary to make sure what is optimal. As long as the system can shorten the time of moving from the non-optimum to the border and from the border to the optimum, the system is satisfactory [9].

The traditional self-organization theories discuss the system level problems under the condition of free borders and fixed borders, which actually reflect subjective and objective restrictions. Whatever the subjective and objective restrictions are, the satisfaction level of a system under non-optimum conditions is called meso-optimum. In the actual analysis, 
under certain optimal interval of aims and results, the premise is to find out the correspondent non-optimum interval. Thus, a meso-optimum interval is decided by the optimum and nonoptimum interval and if the results of decisions fall in this interval, we can call them trusted meso-optimum. All systems have optimum and non-optimum attribute if the optimum attributes of the system can be distinguished and the nonoptimum attributes of the system can be controlled, moreover, the goal of the system can be selection, then the system goal exists in a maximize the satisfaction. [11]

Definition 24 Let $\lambda$ is a parameter of $f(v(o, 0))$ in factor space, and $\lambda=\left\{\lambda_{1}, \cdots, \lambda_{n}\right\}$, then

$$
v^{\lambda}(o, 0)=\left\{v\left(o_{i}, 0\right) \geq \lambda: 0 \leq \lambda \leq 1, o_{i} \in O, i=1, \cdots, n\right\}
$$

is called the $\lambda$ level of positive or is called optimum order ( $\lambda$ order), and $\lambda$ is order parameter.

Definition 2.5 Let $\eta$ is a parameter of $f(v(0, \bar{o}))$ in factor space, and $\eta=\left\{\eta_{1}, \cdots, \eta_{n}\right\}$, then

$$
v^{\eta}(0, \bar{o})=\left\{v\left(0, \bar{o}_{i}\right) \leq \eta:-1 \leq \eta \leq 0, \bar{o}_{i} \in \bar{O}, i=1, \cdots, n\right\}
$$

is called $\eta$-level of negative or is called non-optimum $\operatorname{order}(\eta$ order $)$, and $\eta$ is a unordered parameter.

Definition 2.6 Let $\lambda-\eta$ is a parameter of $f(v(o, \bar{o}))$ in factor space,

$$
\lambda-\eta=\left\{\lambda_{1}-\eta_{1}, \lambda_{2}-\eta_{2} \cdots, \lambda_{n}-\eta_{n}\right\}
$$

Then

$$
\begin{aligned}
& v^{\lambda-\eta}\left(o_{i}, \bar{o}_{i}\right)=\left\{\eta \leq v\left(o_{i}, \bar{o}_{i} \leq \lambda: \lambda>0, \eta<0\right.\right. \\
& \left.o_{i} \in O, \bar{o}_{i} \in \bar{O}, i=1, \cdots, n\right\}
\end{aligned}
$$

is called the $\lambda-\eta$ level of meso-optimum effects or is called meso-optimum order ( $\lambda-\eta$ order), and $\lambda-\eta$ is an order parameter of the system self-organizing.

We will be called extension order about synthesize $\lambda$ order, $\eta$ order and $\lambda-\eta$ order, that is, $\{\lambda, \eta, \lambda-\eta\}$ order.

Definition 2.4 belongs to the issue of the traditional selforganizing, and it is a core issue that how to determine $\lambda$ of factor-order space, thus there is a time limitation on the system's stay in the optimum category. Within a certain time, because the system is stable, it stays in the optimum category. Definition 2.5 shows that, when the factors of the system cannot determine the optimum attributes, this system belong to the category of the non-optimum, then the function of the selforganization system is how to determine the minimum nonoptimum. Definition 2.6 shows that there are the optimum and non-optimum attributes to everything, and the final direction of the system can be only achieved through practice and the transition of the optimum and non-optimum attributes. The state of the system decides its goal by choosing between optimum and non-optimum attributes. [12]

According to definition 2.4-2.6, a factor-order of the selforganizing has three factor characteristics, that is, the factororder of the positively level

$$
A_{\lambda}=\left(F, c(o, 0), v^{\lambda}(o, 0)\right),
$$

the factor-order of the negatively level

$A_{\eta}=\left(F, c(0, \bar{o}), v^{\eta}(0, \bar{o})\right)$

and the factor-order of the neutrally level

$$
A_{\lambda-\eta}=\left(F, c(o, \bar{o}), v^{\lambda-\eta}(o, \bar{o})\right) \text {. }
$$

Thus, the factor-order of the self-organizing can be as the following formulation:

$$
A=\left(\begin{array}{ccc}
F & c(o, 0) & v^{\lambda}(o, 0) \\
c(0, \bar{o}) & v^{\eta}(0, \bar{o}) \\
c(o, \bar{o}) & v^{\lambda-\eta}(o, \bar{o})
\end{array}\right)
$$

In the analysis of the self-organizing system, to some extent, the optimum and non-optimum attributes can be judged and controlled based on experience and intuition. In fact, the optimum and the non-optimum attributes depends on recognition degree in self-organizing process of system. Some factor-order of system are both optimum and non-optimum attributes. For instance, there is acceptable and unacceptable aspect when the decision is made. At the same time, there exist satisfactory results, as well as unsatisfactory ones, et al. The uncertainty decision arises during balancing those optimum and non-optimum attributes [13].

\section{SELF-ORGANIZATION BASED ON EXTENDED ENTROPY}

\section{A. Extended Order Based on Meso-optimum}

The key to solving self-organizing level is the study of properties about factor-order of the system, from which the model of self-organization system is formed. In the course of solving self-organizing level, we must come out of the habitual domain; open up systems involved in the problems and put forward creative methods. In fact, a system factor-order with meso-optimum in self-organization system, which is including the maximum optimum order and the minimum non-optimum order, the minimum optimum order and maximum nonoptimum order. The above meso-optimum, which makes it possible to open up systems from different viewpoints, is the basis for both meso-optimum thinking and solving selforganizing level.

The study on the self-organizing level of systems helps us understand the optimum and non-optimum between different parts more clearly. The theory of system self-organizing has given a kind of description of the order structure of systems, which studies systems from the composition and the relationship between optimum attributes and non-optimum 
attributes of system factors. The analysis on lots of system has shown that the order structure of systems can be studied from meso-optimum nature of system factors. From the view of meso-optimum nature of system factors, a system factor can be divided into two attributes: optimum attributes and nonoptimum attributes. It is saying that the optimum attributes are the base, and the non-optimum attributes are what we used. Every system is the entity of the optimum and the nonoptimum attributes.

Thus, every factor, and every system can be seen as factororder with goals and meso-optimum order aiming to reach those system goals. The meso-optimum order of the factororder can affect (positively, negatively, or neutrally) the fulfillment of the goals of other factor-order, thereby establishing a relation. The measurement of the system's goals of a factor-order can be represented by using meso-optimum $\operatorname{order} v(o, \bar{o})$ based on optimum and non-optimum attributes.

The attainability of the objective of the system shows that the distance between the recognized goal of the system and the actual goal of the system is acceptable. The achievability of the function of the system refers that the actual functional resources are near to the objective-required resources. The controllability of the environment of the system refers to the self-organizing capacity or the order parameters achieving the permitted value. [14]

In the self-organization system, every factor and every system, can be seen as extended factor-order $A=(F, c, v)$ with goals and behaviors aiming to reach those goals. The characteristics of factor-order can affect (positively, negatively and neutrally) the fulfillment of the goals of other factor-order, thereby establishing a relation. The satisfaction or fulfillment of the goals of a factor-order can be represented using the set of the extended order parameter, which is:

$$
\sigma=\left\{\sigma^{\lambda}, \sigma^{\eta}, \sigma^{\lambda-\eta}\right\}=\left\{v^{\lambda}(o, 0), v^{\eta}(0, \bar{o}), v^{\lambda-\eta}(o, \bar{o})\right\} .
$$

Relating this to the higher, the satisfaction of a system $\sigma_{\text {sys }}$ can be recursively represented as a function

$$
f_{j}: v(o, \bar{o}) \rightarrow[-1,1]\left(f_{j} \in F, j=1, \cdots, m\right)
$$

And, $E=\left\{e_{1}, \cdots, e_{n}\right\}$, thus

$$
\begin{aligned}
& \sigma_{s y s}=f_{j}\left(\sigma_{s y s}^{\lambda}, \sigma_{s y s}^{\eta}, \sigma_{s y s}^{\lambda-\eta}\right) \\
& =f_{j}\left(v^{\lambda}(o, 0), v^{\lambda-\eta}(o, \bar{o}), v^{\eta}(0, \bar{o}),\right. \\
& \left.W\left(w^{\lambda}, w^{\eta}, w^{\lambda-\eta}\right)\right)
\end{aligned}
$$

We have:

$$
\begin{aligned}
& \sigma_{s y s}^{\lambda}=f_{j}\left(\sigma_{1}^{\lambda}, \sigma_{2}^{\lambda}, \cdots, \sigma_{n}^{\lambda}, w_{1}^{\lambda}, w_{2}^{\lambda}, \cdots, w_{n}^{\lambda}\right) \\
& =f_{j}\left(v_{1}^{\lambda}(o, 0), v_{2}^{\lambda}(o, 0), \cdots, v_{n}^{\lambda}(o, 0), w_{1}^{\lambda}(o, 0),\right. \\
& \left.w_{2}^{\lambda}(o, 0), \cdots, w_{n}^{\lambda}(o, 0)\right)
\end{aligned}
$$

$$
\begin{aligned}
& \sigma_{s y s}^{\eta}=f_{j}\left(\sigma_{1}^{\eta}, \sigma_{2}^{\eta}, \cdots, \sigma_{n}^{\eta}, w_{1}^{\eta}, w_{2}^{\eta}, \cdots, w_{n}^{\eta}\right) \\
& =f_{j}\left(v_{1}^{\eta}(0, \bar{o}), v_{2}^{\eta}(0, \bar{o}), \cdots, v_{n}^{\eta}(0, \bar{o}), w_{1}^{\eta}(0, \bar{o}),\right. \\
& w_{2}^{\eta}(0, \bar{o}), \cdots, w_{n}^{\eta}(0, \bar{o}) \\
& \sigma_{s y s}^{\lambda-\eta}=f_{j}\left(\sigma_{1}^{\lambda-\eta}, \sigma_{2}^{\lambda-\eta}, \cdots, \sigma_{n}^{\lambda-\eta}, w_{1}^{\lambda-\eta}(o, \bar{o})\right. \\
& \left.w_{2}^{\lambda-\eta}(o, \bar{o}), \cdots, w_{n}^{\lambda-\eta}(o, \bar{o})\right) \\
& =f_{j}\left(v_{1}^{\lambda-\eta}(o, \bar{o}), v_{2}^{\lambda-\eta}(o, \bar{o}), \cdots, v_{n}^{\lambda-\eta}(o, \bar{o}),\right. \\
& \left.w_{1}^{\lambda-\eta}(o, \bar{o}), w_{2}^{\lambda-\eta}(o, \bar{o}), \cdots, w_{n}^{\lambda-\eta}(o, \bar{o})\right) \\
& \text { where } \quad w_{i}^{\lambda}(o, 0), w_{i}^{\eta}(0, \bar{o}), w_{i}^{\lambda-\eta}(o, \bar{o})(i=1,2, \cdots, n)
\end{aligned}
$$

are determined tautologically by the importance of $\sigma_{i}^{\lambda}$, of $\sigma_{i}^{\eta}$, and of $\sigma_{i}^{\lambda-\eta}$ of each factor to the satisfaction of the systems $\sigma_{\text {sys }}^{\lambda}, \sigma_{\text {sys }}^{\eta}$ and $\sigma_{\text {sys }}^{\lambda-\eta}$.

\section{B. Measurement of Self-organization Level}

\section{1) $\eta$ order and $\eta$ entropy}

In reality, every uncertain system belongs to the nonoptimum category. It meets the recognition and realization of mankind to analyze the causes of non-optimum category and the ways to reach optimum from the viewpoint of the nonoptimum category. According to the concept of the factor-order analysis, when $\sigma=v^{\eta}(0, \bar{o}) \in[-1,0]$, we have

$$
\begin{aligned}
& \sigma_{s y s}^{\eta}=f_{j}\left(\sigma_{1}^{\eta}, \sigma_{2}^{\eta}, \cdots, \sigma_{n}^{\eta}, w_{1}^{\eta}, w_{2}^{\eta}, \cdots, w_{n}^{\eta}\right) \\
& =f_{j}\left(v_{1}^{\eta}(0, \bar{o}), v_{2}^{\eta}(0, \bar{o}), \cdots, v_{n}^{\eta}(0, \bar{o}),\right. \\
& w_{1}^{\eta}(0, \bar{o}), w_{2}^{\eta}(0, \bar{o}), \cdots, w_{n}^{\eta}(0, \bar{o})
\end{aligned}
$$

The satisfaction of this system $\sigma_{\text {sys }}^{\eta}$ belongs to the issue of the self-organizing in the non-optimum category, where $w_{i}^{\eta}(0, \bar{o})$ is determined tautologically by the share of $\sigma_{i}^{\eta}$ ( $\sigma_{i}^{\eta}=v_{i}^{\eta}(0, \bar{o})$, the characteristics values of the nonoptimum) of each factor to the satisfaction of the systems $\sigma_{\text {sys }}^{\eta}$. Thus, we can be design a entropy of the non-optimum (or is called $\eta$ entropy), that is

$$
H\left(P\left(\sigma_{\text {sys }}^{\eta}\right)\right)=-\sum_{i=1}^{n} P\left(w_{i}^{\eta} \sigma_{i}^{\eta}\right) \log P\left(w_{i}^{\eta} \sigma_{i}^{\eta}\right)
$$

The goals of the system self-organizing are to achieve minimum $\eta$ entropy in the non-optimum category.

\section{2) Extended entropy based on $\lambda-\eta$ order}

The groundwork of the self-organization theory of the system is the systematic non-optimum analysis doctrine. For any uncertain system, whether it has entered the non-optimum category or gone out of non-optimum category are judged 
through factor-order. In order to hold the system in the optimum category under certain degree and stage, we have to recognize and control the non-optimum attributes of the system factor through extended order. As we know, the non-optimum attributes of the system factor are not only dynamic, but also evaluative. In order to measure the degree of the evolution of the system's meso-optimum, the criteria of evolution have to be set up (meso-optimum criteria). According to the concept of the factor-order space, when $\sigma=v^{\lambda-\eta}(o, \bar{o}) \in[-1,1]$, we have

$$
\begin{aligned}
& \sigma_{s y s}^{\lambda-\eta}=f_{j}\left(\sigma_{1}^{\lambda-\eta}, \sigma_{2}^{\lambda-\eta}, \cdots, \sigma_{n}^{\lambda-\eta}, w_{1}^{\lambda-\eta}(o, \bar{o})\right. \\
& \left.w_{2}^{\lambda-\eta}(o, \bar{o}), \cdots, w_{n}^{\lambda-\eta}(o, \bar{o})\right) \\
& =f_{j}\left(v_{1}^{\lambda-\eta}(o, \bar{o}), v_{2}^{\lambda-\eta}(o, \bar{o}), \cdots, v_{n}^{\lambda-\eta}(o, \bar{o}),\right.
\end{aligned}
$$

$$
\left.w_{1}^{\lambda-\eta}(o, \bar{O}), w_{2}^{\lambda-\eta}(o, \bar{o}), \cdots, w_{n}^{\lambda-\eta}(o, \bar{O})\right)
$$

The satisfaction of this system $\sigma_{\text {sys }}^{\eta}$ belongs to the issue of the self-organizing in the optimum category and non-optimum, where $w_{i}^{\lambda-\eta}(o, \bar{O})$ is determined tautologically by the share of $\sigma_{i}^{\lambda-\eta}\left(\sigma_{i}^{\lambda-\eta}=v_{i}^{\lambda-\eta}(o, \bar{O})\right.$, the characteristics values of the optimum and non-optimum, at the same time) of each element to the satisfaction of the systems $\sigma_{s y s}^{\lambda-\eta}$. Thus, we can be design extension entropy of the optimum and non-optimum (or is called $\lambda-\eta \quad$ entropy), that is

In the (2), the satisfaction of this system $\sigma_{s y s}^{\lambda}$ belongs to the issue of the traditional self-organizing [14], where is $w_{i}^{\lambda}$ determined tautologically by the share of $\sigma_{i}^{\lambda}$ $\left(\sigma_{i}^{\lambda}=v_{i}^{\lambda}(o, 0)\right.$, the characteristics values of the optimum) of each factor to the satisfaction of the $\sigma_{\text {sys }}^{\lambda}$.

The extended entropy is useful because it gives an analysis method of the factor-order representation for the selforganization level of the system, which is measurement of an uncertain system. An extended order would assume that the extended entropy of the factors of a system would be also extended entropy of the self-organization level of the system. However, this is not always the case, since some order parameters can "take advantage" of other order parameters. Thus, we need to concentrate also on the cooperation of the factor-order. [15]

\section{Measurement of Self-organization Level}

If the self-organization level of a system considers more than two levels, then $\sigma_{\text {sys }}^{\lambda-\eta}$ of higher levels will be recursively determined by the extended entropy of lower levels. However, $f_{i}$ 's most probably will be very different on each level. Certainly, an important question remains: how do we determine the function $f_{i}$ and the extended order parameter? To this question, there is no complete answer. One option would be to approximate numerically $f_{i}$. An explicit $f_{i}$ may be difficult to find, but an approximation can be very useful. Another method consists of function the system: removing or altering factors of the system, and observing the effect on $\sigma_{\text {sys }}^{\lambda-\eta} \cdot[16]$
Through analyzing the effects of different lesions, the function $f_{i}$ can be reconstructed and the $\{\lambda, \eta, \lambda-\eta\}$ obtained. If $\Delta\left(w_{i}^{\lambda} \sigma_{i}^{\lambda}-w_{i}^{\eta} \sigma_{i}^{\eta}\right)$ is a small change in any $\sigma^{\lambda}$ produces $\left|\Delta \sigma_{\text {sys }}^{\lambda-\eta}\right| \geq\left|\Delta \sigma_{i}^{\lambda}\right|+\left|\Delta \sigma_{i}^{\eta}\right|$, the system can be said to be the level of the satisfaction. What could then be done to maximum $\sigma_{s y s}^{\lambda-\eta}$ ? How can we relate $\sigma_{i}^{\lambda-\eta}$,s and avoid conflicts between factors? This is not an obvious task, for it implies bounding the factor-order's characteristics that reduce other $\sigma_{i}^{\eta}$ 's while preserving their functionality. [17]

Not only should the optimum or the non-optimum between $\Delta\left(w_{i}^{\lambda} \sigma_{i}^{\lambda}-w_{i}^{\eta} \sigma_{i}^{\eta}\right)$ of factors be minimized, but the synergy or "positive interference" should also be promoted. Dealing with complex systems, it is not feasible to tell each factor what to do or how to do it, but their behaviors need to be constrained or modified so that their goals will be reached, blocking the goals of other factors as little as possible. These constraints can be called mediators. They can be imposed from the top down, developed from the bottom up, be part of the environment, or be embedded as an aspect of the system. Mediators are determined by an observer, and can be internal or external to the system (depending on where the sub-optimum sets the boundaries of the system) (He Ping, 2011).

Confusion may arise when people describe systems as the lower level causing change (that is $w_{i}^{\lambda} \sigma_{i}^{\lambda} \leq w_{i}^{\eta} \sigma_{i}^{\eta}$ ) in the emergent properties of the same system. Vice versa, downward causation is the idea that higher level properties (that is $\left.w_{i}^{\lambda} \sigma_{i}^{\lambda} \geq w_{i}^{\eta} \sigma_{i}^{\eta}\right)$ constrain or control components at the $\eta$ order. Speaking about causality between $\lambda$ order and $\eta$ order is not accurate, in fact, they are dynamic. What we could say is 
that when we observe certain conditions in the $\eta$ order (lower level), we can expect to observe certain properties at the $\lambda$ order (higher level) and vice versa. There is correlation function, but not actual causation.

\section{CONCLUSION}

Factor space theory is a new theory of Information Science and System Science, and the basic principle of the system nonoptimum science is to discuss the choice of uncertainty from the crossing perspective between optimum and non-optimum. Based on the theory of space factor, this thesis adopts the use of non-optimum to explore system self-organization, and proposes several new concepts about system self-organization by applying factor-order into the discussion of system uncertainty. The research shows that the system factor space establishes the information system, and the factor-state of optimum and non-optimum attribute is an effective parameter to describe the system ordering. The key to self-organization of uncertain system is the realization of the two mappings, and the transformation of disorder and order can be achieved by factor mapping and object inversion. The research content of this paper will get further development in the following several aspects: Firstly, how to find factor of object is an important part of the study of factor space in the design and operation of the system. It should be made clear that the premise of system ordering and optimization is not to find knowledge, but factor. Because only after the finding of factor can find knowledge. Secondly, factor-order is the key to study optimum and nonoptimum. In the study, it is necessary to introduce the humancomputer interaction algorithm to solve the problem between the perception, and how to effectively improve the data analysis and judgment, collaborative perception is also a research subject.

\section{ACKNOWLEDGMENTS}

The authors are grateful for the support given by National Natural Science Foundation of China (Grant No. 61272170). We also thank reviewers for insightful and helpful suggestions.

\section{REFERENCES}

[1] P Jaroslaw, P Filip, S Tomasz, Theoretical model for mesoscopic-level scale-free self-organization of functional brain networks, IEEE Transactions on Neural Networks, 2010, vol.21,no.11, pp.47-58.
[2] K Nobuhiko, T Shoji, S Yasuyuki, Establishment of self-organization system in rapidly formed multicellular heterospheroids, Biomaterials, 2011, vol.32, no.26,pp.6059-6067.

[3] He Ping, Theories and Methods of Non-optimum Analysis on Systems, Journal of Engineering Science, 2004, vol.2, no. 1, pp. 73-80.

[4] He Ping, Risk Assessment of Network Security Based on Non-optimum Characteristics Analysis, International Journal of Advanced Computer Science and Applications, 2013, vol.4, no.10, pp.73-79.

[5] Ping He, System Non-optimum Analysis and Extension Optimum Theory, In: Guangya Chen, ed, Proc. of the Int'l conf on Systems Science and Systems Engineering, 2003, pp.131-137.

[6] He Ping, Method of System Non-optimum Analysis in Crisis Management, Proc. of Second International Conference of Information System for Crisis Response and Management, 2007, pp. 640-645.

[7] Ping He. Maximum Sub-Optimum Decision-Making Based on NonOptimum Information Analysis. Advanced Science Letters, 2012, vol.5, no.1, pp. 376-385.

[8] Ping He. Characteristics Analysis Of Network Non-Optimum Based On Self-Organization Theory. Global Journal of Computer Science and Technology, 2010, vol.10, no.9, pp.62-77.

[9] Wang Peizhuang, Factor spaces and data science, Journal of Liaoning Technical University (Natural Science) , 2015, vol.34, no.1, pp.273280.

[10] Ping He, Kaiqi Zou, Fuzzy Meso-optimum Sets and Trusted Optimum Analysis, ICIC EXPRESS LETTERS, Part B: Applications, 2015,vol.6, no.8, pp.2079-2086.

[11] Weidong Tao, Studies of human-computer interaction system based on trust intuition learning theory, $20102^{\text {nd }}$ International Conference on Computer Engineering and Technology, 2010, pp.455-459

[12] Jiantong He, A New Intelligence Analysis Method Based on Suboptimum Learning Model, 2009 ETP International Conference on Future Computer and Communication, 2009, pp.116-119.

[13] Zengtang Qu, Sub-optimum evaluation on incomplete network information system, 2010 International Conference on E-Health Networking Digital Ecosystems and Technologies (EDT), 2010, pp.465468.

[14] HJ Morowitz, CHAPTER V-The Second Law of Thermodynamics, Cuad Geogr, 2014, vol.23, no.1, pp.109-124.

[15] ET Jaynes, On the rationale of maximum-entropy methods, Proceedings of the IEEE, 2010, vol.70, no.9, pp.939-952.

[16] S Cano-Andrade, GP Beretta, MRV Spakovsky, Steepest-entropyascent quantum thermodynamic modeling of decoherence in two different microscopic composite systems, Phys.rev.a, 2015, vol.17, no.3, pp. 57-73, 2015.

[17] A García-Hiernaux, J Casals, M Jerez, Estimating the system order by subspace methods, Computational Statistics, 2012, vol.27, no.3, pp.411425 . 